\title{
Derivation of a wear scar geometry-independent coefficient of friction from fretting loops exhibiting non-Coulomb frictional behaviour
}

\author{
X. Jin, W. Sun, P.H. Shipway ${ }^{1}$ \\ Faculty of Engineering, University of Nottingham, Nottingham, UK
}

\begin{abstract}
One source of variation of the sliding tractional force in a gross-slip fretting cycle is the geometrical interaction of the developing wear scars on the opposing specimens. An existing model has been developed to include the compliance of the fretting test apparatus. It has thus been demonstrated that through the influence on the tractional force, the geometrical development of the wear scars affects the slip amplitude and the dissipation of frictional energy in each loop. A method to determine a coefficient of friction which is independent of system stiffness and developments in the geometry of the wear scars is proposed.
\end{abstract}

Keywords: friction, ECoF, fretting loop, wear scar

\section{Introduction}

Fretting is the small amplitude oscillation between two bodies in contact which occurs in a wide variety of mechanical systems. Although the amplitude of fretting is small $(<300 \mu \mathrm{m})$, fretting can cause serious local damage of the material through wear and fatigue [1]. Fretting fatigue usually occurs when the displacement amplitude is small and there is no relative slip between the contact surfaces for part of the contact. This condition is known as stick-slip. Fretting wear, on the other hand, becomes the major cause of fretting damage when the displacement amplitude is large enough for the whole contact area to experience relative slip, which is known as gross sliding. The identification of these different fretting conditions is usually performed by observing the shape of the fretting loop which is the plot of the tractional force against the corresponding displacement; Vingsbo and Söderberg [1] first identified that in stick-slip, the fretting loop would be elliptical in shape whereas in gross sliding, the loop would open up to become parallelogram-like.

Figure 1a shows an idealised schematic fretting loop for fretting in gross sliding. The relative displacement between the bodies is represented by the symbol $\Delta$, and the corresponding tractional force is $Q$. It should be noted that $\Delta$ is measured remotely from the contact itself, and is not the same as the slip in the contact; the slip amplitude $\left(\delta^{*}\right)$ is smaller than the applied displacement amplitude $\left(\Delta^{*}\right)$ as there is compliance in the system, associated with elastic deformation of the specimen, the fixture and the test rig. Together, these can be represented via a system stiffness, $S$, as indicated in Figure 1a as the slope of the sides of the fretting loop. The actual slip amplitude, $\delta^{*}$, cannot easily be measured directly but can be determined from the fretting loop as the displacement amplitude at zero tractional load (see Figure 1). The area inside the loop represents the energy dissipated $\left(E_{d}\right)$ by frictional work in the cycle [2]. Alongside the development of the wear scar itself (in terms of its size and nature), the tractional force is commonly used to characterise the behaviour of the contact;

\footnotetext{
${ }^{1}$ Corresponding author
} 
evolution of this tractional force is an important tool in that it can be measured throughout the test, whereas the scar characterisation can only be performed at the end of the test.

(a)

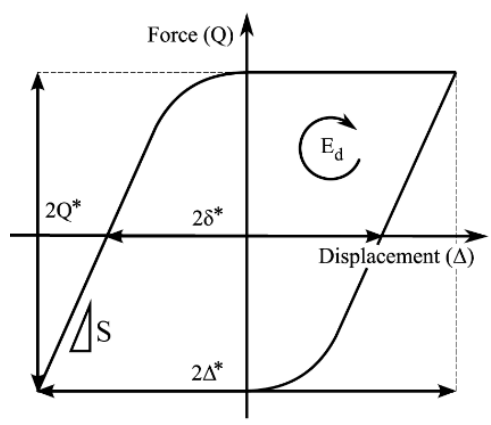

(b)

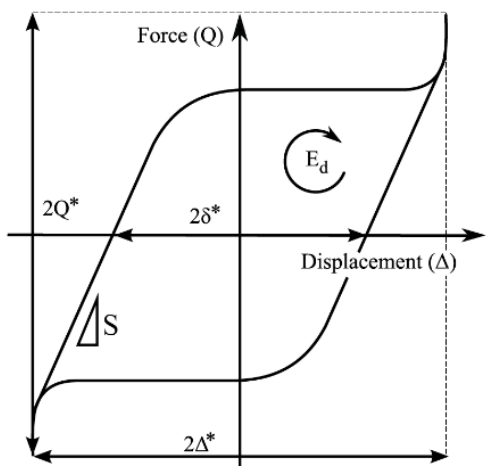

(c)

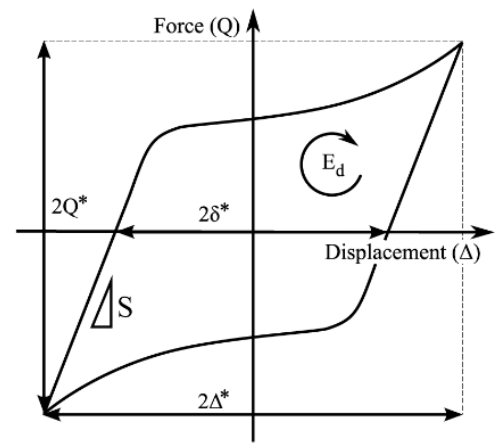

Figure 1 Schematic diagram of (a) idealised fretting loop (b) fretting loop with high tractional force peak at the ends of gross sliding (c) fretting loop with hook-like increase in tractional force during gross sliding; in all cases, terms are defined in the text.

To allow comparisons between tests conducted under different loading conditions, measures of the tractional force during sliding are often normalised against the applied force; this ratio of $Q / P$ is commonly described as the coefficient of friction $(\mathrm{CoF})$. However, despite its importance, there is no general consensus regarding the definition of the $\mathrm{CoF}$ in a fretting test. The $\mathrm{CoF}$ is often defined as the ratio of the maximum tractional force $\left(Q^{*}\right)$ within a cycle to the applied normal load $(P)$. This definition is appropriate for a fretting loop with a shape close to that of the ideal (Figure 1a); here, the flat top and bottom indicate that the dynamic tractional force only depends on the applied load and the surface conditions according to Coulomb's friction law [3]. However, it has been reported by many researchers [4-8] that the tractional force changes significantly across the stroke (i.e. the top and bottom of the loop are not flat); it has been argued that this behaviour is more prevalent with ductile materials [9]. Such non-Coulomb frictional behaviour is typically observed in two main ways: (i) it appears as a very high peak in the tractional force only at the ends of the stroke (i.e. top right and bottom left of the loop as shown in Figure 1b) [9,10]; (ii) the tractional force increases during the whole gross sliding stage and reaches the maximum at the ends of the stroke which resembles a hooklike feature (see Figure 1c) [11,12]. In either of these circumstances, the validity of employing the maximum tractional force $\left(Q^{*}\right)$ to calculate the $\mathrm{CoF}$ is questionable.

In response to this issue, it was first suggested by Fouvry et al. [9] that the peaks in tractional force at the motion extremes in a fretting cycle (Figure 1b) are caused by the physical interaction between the edges of the wear scar; they termed this the ploughing effect. Ploughing usually occurs during fretting between ductile materials where material build-up due to bulk plastic flow occurs at the wear scar edges. This has been further supported by Dick et al. [10], who observed significant material build up at the edges of the wear scar with a cylinder-on-flat configuration, and built a finite element model which successfully modelled the increase in tractional force at the ends of the fretting stroke. Mulvihill et al. [11] argued that it may not just be the interaction between the edges but also the interaction between specific regions within the contact that cause the tractional force to rise in this way. To minimise the influence of the ploughing effect in calculating the CoF, Fouvry et al. [9] proposed the concept of the energy coefficient of friction $(\mathrm{ECoF}), \mu_{E}$. This method uses the energy 
dissipation over a fretting loop $\left(\mathrm{E}_{\mathrm{d}}\right)$ and the slip amplitude $\left(\delta^{*}\right)$ to calculate an averaged $\mathrm{CoF}$ as follows:

$$
\mu_{E}=\frac{E_{d}}{4 P \delta^{*}}
$$

Whilst being attractive in its simplicity, this method simply provides a method for deriving an average $\mathrm{CoF}$, and does not seek to separate the observed $\mathrm{CoF}$ into its various physical contributions, namely (i) the contact $\mathrm{CoF}$ and (ii) geometrical effects. In this regard, Mulvihill et al. [11] developed a simplified mechanical model of geometrical interactions which would serve to aid in the development of this understanding. They presented a one-degree-of-freedom wear-scar interaction model with a point contact between a rigid round-ended body and a groove (the groove represented the profile that develops in a specimen pair due to the process of fretting wear). By oscillating the round-ended body, they successfully predicted fretting loops with a variation in the tractional force across the whole gross sliding stage (as shown schematically in Figure 1c); they were thus able to argue robustly that it is the physical slope of the wear scars (and the associated normal displacement of the two bodies relative to each other) which causes the variation in tractional force over the contact slip. Experimental evidence to support this concept has recently been provided by Hintikka et al. [12] who measured the displacements between the specimens normal to the fretting motion. They found that when non-Coulomb friction behaviour is observed, specimens are also observed to exhibit cyclic motion normal to the fretting direction and demonstrated that the effective gradient of the contact (defined as the ratio of the normal displacement amplitude and the slip amplitude) was related to the magnitude of the non-Coulomb effect (i.e. the deviation of the shape of the fretting loop from the ideal Coulomb-type loop illustrated in Figure 1a towards that illustrated in Figure 1c) [12]. Furthermore, Mulvihill et al. [11] demonstrated that the energy dissipated in a fretting loop $\left(E_{d}\right)$ where geometrical features were resulting in non-Coulomb behaviour would be greater than that dissipated in a loop with no geometrical factors influencing the tractional force, and thus that the ECoF so derived has a dependence on the geometry of the wear scar; as such, it is clear that further development is required to provide a method which allows a geometry-independent $\mathrm{CoF}$ to be derived from a fretting loop.

In the model of Mulvihill et al. [11], the systems making up the fretting contact are rigid, leading to the applied displacement amplitude $\Delta^{*}$ and the slip amplitude $\delta^{*}$ being identical. However, if the compliance of the rig is considered (which means that $\Delta^{*}>\delta^{*}$ ), it is clear that an increase in the tractional force associated with geometrical developments of the scar also leads to a reduction in the slip amplitude [13]. In most experimental fretting apparatus, it is the displacement amplitude (rather than the slip amplitude) which is measured and controlled, and thus a method which addresses the effects of geometrical changes on the $\mathrm{CoF}$ derived from a fretting loop measured in a fretting rig with a system compliance is required. In the current work, the wear-scar interaction model of Mulvihill et al. [11] will be extended by including the compliance of the system in the model. Analysis will be performed to examine the effect of the shape of the wear scar and the compliance of the system on the slip amplitude and the ECoF when a constant displacement amplitude is applied. Most significantly, an improved method for deriving the geometry-independent CoF from a gross-slip fretting loop will be proposed. 


\section{Development of the modified wear scar interaction model}

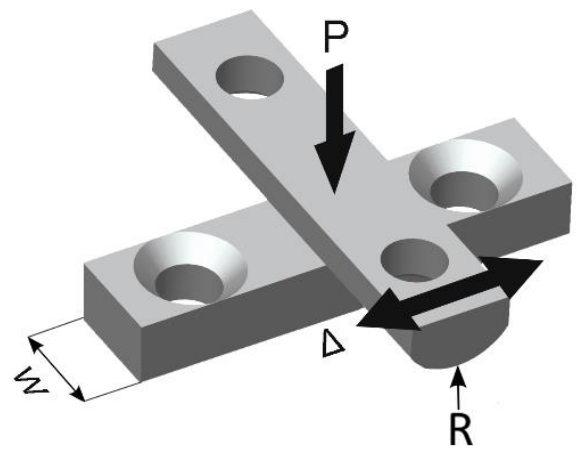

Figure 2 Cylinder-on-flat specimen arrangement employed in fretting tests [14]

(a)

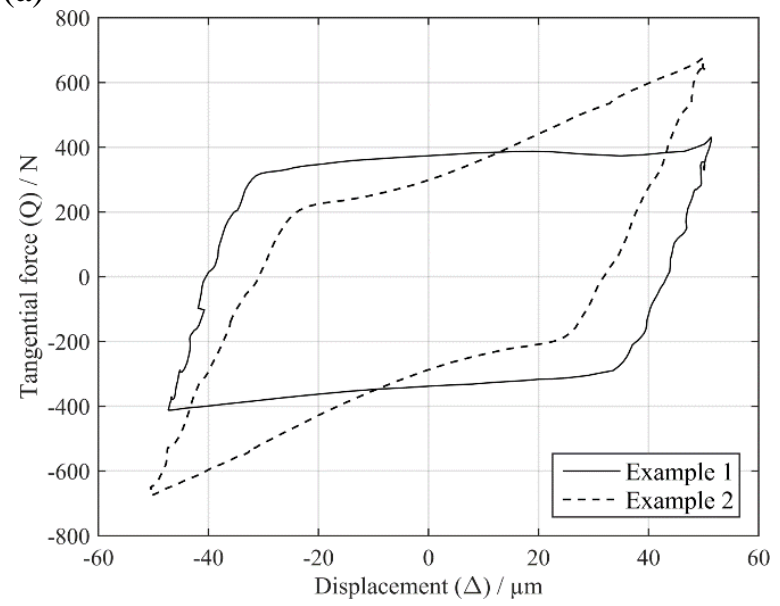

(b)

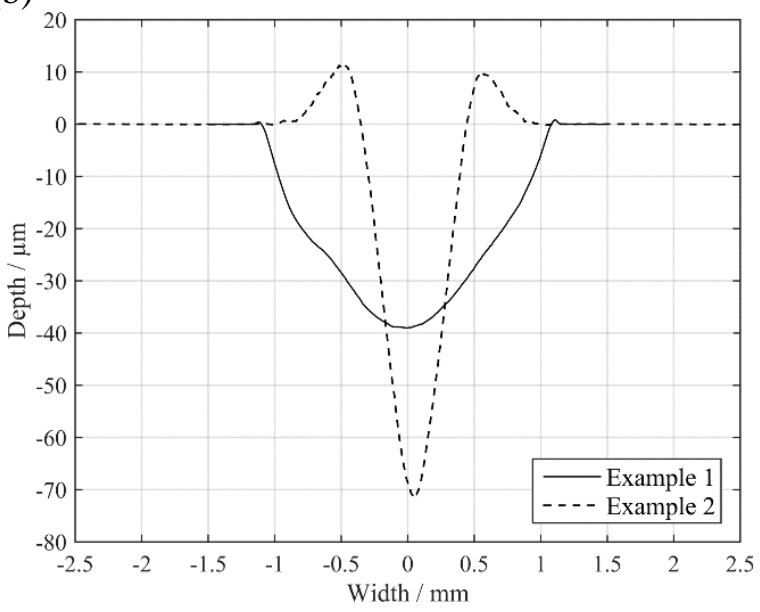

Figure 3 Examples of loops and wear scar profiles from cylinder-on-flat fretting tests illustrating behaviour as shown in Figure 1a and Figure 1c. In both cases, $P=450 \mathrm{~N}, \Delta^{*}=50 \mu \mathrm{m}$, cycles $=500$ 000, frequency $=20 \mathrm{~Hz}$ : (a) measured fretting loops from the end of the test; (b) wear scar profiles from the flat specimen.

Many experimental researchers into fretting utilise con-conforming contact geometry, typically a sphere-on-flat or a cylinder-on-flat geometry. At the University of Nottingham, the bulk of our work has utilised the latter, often with a $6 \mathrm{~mm}$ radius cylinder. Specimens are assembled in a cylinder-onflat arrangement (Figure 2) which generates a line contact $(W)$ of $10 \mathrm{~mm}$ in length (see the work of Warmuth et al. for a detailed description of the test apparatus [14]). A normal load, $P$, is applied to the upper (moving) specimen through a dead-weight with the fretting motion being applied perpendicular to the axis of the cylindrical specimen. Fretting loops with different shapes have been observed, with the shapes being dependent upon the materials and test conditions employed. Figure 3 shows measured fretting loops from two fretting tests illustrating the range of behaviour observed; Example 1 exhibits a typical flat-topped loop (similar to the schematic diagram of Figure 1a) whilst Example 2 exhibits a typical hook-shaped loop (similar to the schematic diagram of Figure 1c). It can be seen in Figure 3a that Example 2 exhibits a maximum tractional force which is much larger than the maximum tractional force observed in Example 1. Although the displacement amplitude $\left(\Delta^{*}\right)$ is the 
same for both tests, because of system compliance and this higher tractional force, Example 2 has a lower slip amplitude $\left(\delta^{*}\right)$ than Example 1. Figure 3b shows the comparison of the wear scar profiles on the flat specimen; it can be seen that the scar in Example 2 is much deeper and narrower than that observed in Example 1. These observations align with the conclusion drawn by Mulvihill et al. [11], namely that it is the physical slope of the wear scar which causes the variation in tractional force. However, the change in slip amplitude is associated with the change in elastic deformation associated with the differences in tractional force (associated with the change in slope); this was not accounted for in the model of Mulvihill et al. [11], since they assumed an infinitely stiff system. To understand better the differences in the loop of the type presented in Figure 3, the wear scar interaction model of Mulvihill et al. [11] will be modified with the inclusion of the system stiffness $(S)$.

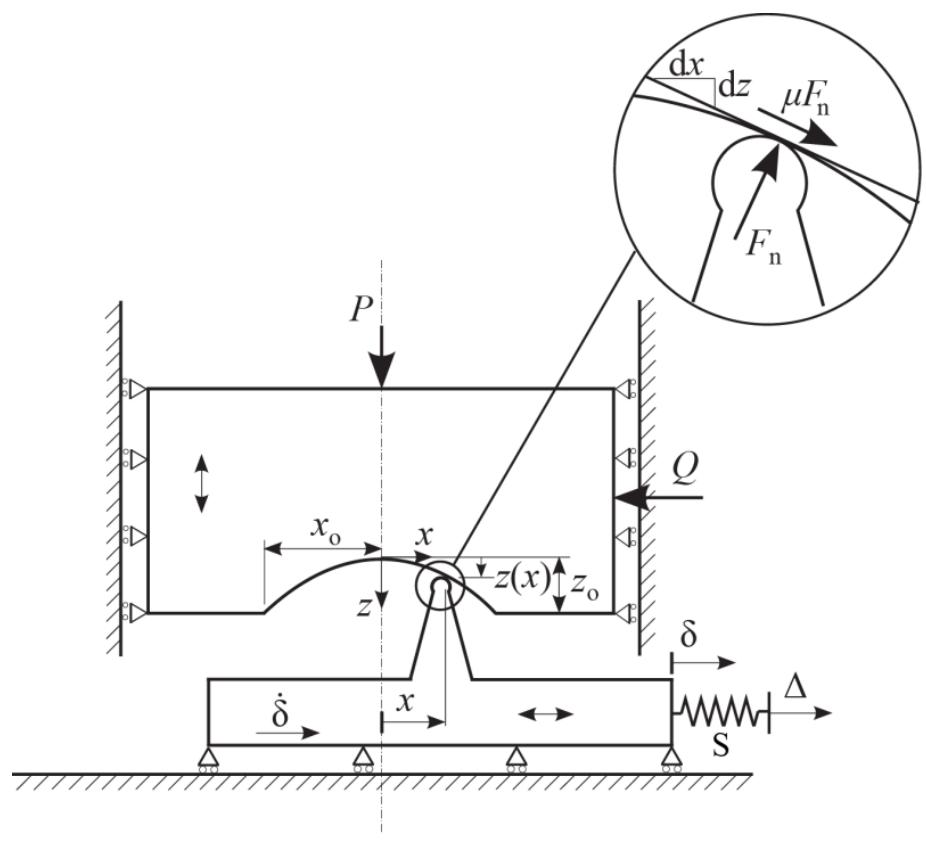

Figure 4 Illustration of wear-scar interaction model with inclusion of the stiffness of the rig (based upon the model of Mulvihill et al. [11]).

To simplify the analysis of the fretting contact, Mulvihill et al. [11] modelled the wear scar interaction as a one-point interaction between two rigid bodies. The rigid bodies both have one degree of freedom (see Figure 4). A vertical force $(P)$ is applied to the pad with the groove which represents the applied load in a fretting test; this pad can only move vertically. The grooved pad is in contact with a roundended pad which can only move horizontally. A spring with stiffness $S$ (representing the stiffness of the system) is attached to the round-ended pad. The oscillatory displacement $\Delta$ (representing the applied displacement in fretting test) is applied to the end of the spring. The resulting displacement of the round-ended pad $(\delta)$ represents the contact slip in fretting. The position of the contact point is $x$ (as indicated in Figure 4). Since both of the pads are themselves rigid, it is observed that:

$$
x=\delta
$$

The shape of the groove is assumed to be parabolic as follows:

$$
z=z_{o}\left(\frac{x}{x_{o}}\right)^{2}
$$


where $z_{o}$ is the depth and $x_{0}$ is the half width of the groove. $x_{o}$ is set to be equal to the displacement amplitude $\left(x_{o}=\Delta^{*}\right)$ and $z_{o}$ represents the displacement normal to the oscillation when $x=x_{o}$. As indicated in Figure 4, the normal force between the two bodies is $F_{n}$ and the corresponding friction force is $\mu F_{n}$, where $\mu$ is the contact $\operatorname{CoF}$ (independent of geometry). Dynamic effects are neglected in this model. Therefore, by applying static force equilibrium, the following equations can be obtained:

$$
\begin{gathered}
\mu F_{n} \frac{d z / d x}{\sqrt{1+(d z / d x)^{2}}} \frac{|\dot{\delta}|}{\dot{\delta}}-F_{n} \frac{1}{\sqrt{1+(d z / d x)^{2}}}=-P \\
\mu F_{n} \frac{1}{\sqrt{1+(d z / d x)^{2}}} \frac{|\dot{\delta}|}{\dot{\delta}}+F_{n} \frac{d z / d x}{\sqrt{1+(d z / d x)^{2}}}=Q
\end{gathered}
$$

In both Equation (4) and (5), $\dot{\delta}$ is the velocity of the round-ended pad. The term $|\dot{\delta}| / \dot{\delta}$ represents the direction of motion of the round-ended pad; when the pad travels in positive direction (from left to right), it will result in a positive friction force on the groove pad (and vice versa). Dividing Equation (5) by Equation (4), $Q$ can be written as:

$$
Q=P \frac{\frac{|\dot{\delta}|}{\dot{\delta}} \mu+d z / d x}{1-\frac{|\dot{\delta}|}{\dot{\delta}} \mu d z / d x}
$$

Differentiation of Equation (3) reveals that:

$$
\frac{d z}{d x}=\frac{2 z_{o}}{x_{o}^{2}} \cdot x
$$

Accordingly, Equation (6) can be rewritten as follows:

$$
Q=P \frac{\frac{|\dot{\delta}|}{\dot{\delta}} \mu+\left(\frac{2 z_{o}}{x_{o}^{2}} \cdot x\right)}{1-\frac{|\dot{\delta}|}{\dot{\delta}} \mu\left(\frac{2 z_{o}}{x_{o}^{2}} \cdot x\right)}
$$

Therefore,

$$
Q= \begin{cases}P \frac{\mu+\left(\frac{2 z_{o}}{x_{o}^{2}} \cdot x\right)}{1-\mu\left(\frac{2 z_{o}}{x_{o}^{2}} \cdot x\right)} & (\dot{\delta}>0) \\ P \frac{-\mu+\left(\frac{2 z_{o}}{x_{o}^{2}} \cdot x\right)}{1+\mu\left(\frac{2 z_{o}}{x_{o}^{2}} \cdot x\right)} & (\dot{\delta}<0)\end{cases}
$$

The relation between the applied displacement $(\Delta)$ and the real slip $(\delta)$ can be formulated as follows: 


$$
\delta=\Delta-\frac{Q}{S}
$$

To model the condition that in most fretting tests the displacement amplitude is controlled, $\Delta^{*}$ is set as a constant in the analysis.

Based on a "U" shaped wear scar profile which is commonly observed in cylinder-on-flat fretting tests (the contact configuration we usually applied in our work at the University of Nottingham). Conversion of the contact conditions in a real wear scar into the round ended point on groove contact interaction model can be achieved via a simple assumption that the ratio of $z_{o} / x_{o}$ is equal to the ratio of the depth of the scar $(D)$ over the half width of the scar $(W)$ as follows:

$$
\frac{z_{o}}{x_{o}}=\frac{D}{W}
$$

Since $x_{o}=\Delta^{*}$, the corresponding groove dimensions $\left(x_{o}\right.$ and $\left.z_{o}\right)$ can be calculated according to Equation (11) for each wear scar.

\section{Effects of system stiffness and groove shape on friction behaviour}

The effects of the system stiffness and the groove shape on the tractional behaviour in fretting will be analysed via the point contact model in this section, with the response being presented in terms of fretting loops. The analysis has been performed with the following parameters which are representative of those employed in our work at the University of Nottingham; displacement amplitude, $\Delta^{*}$, of $50 \mu \mathrm{m}$, normal load, $P$, of $450 \mathrm{~N}$, system stiffness, $S$, of $35 \mathrm{MN} / \mathrm{m}$ and contact $\mathrm{CoF}$, $\mu$, of 0.8 .

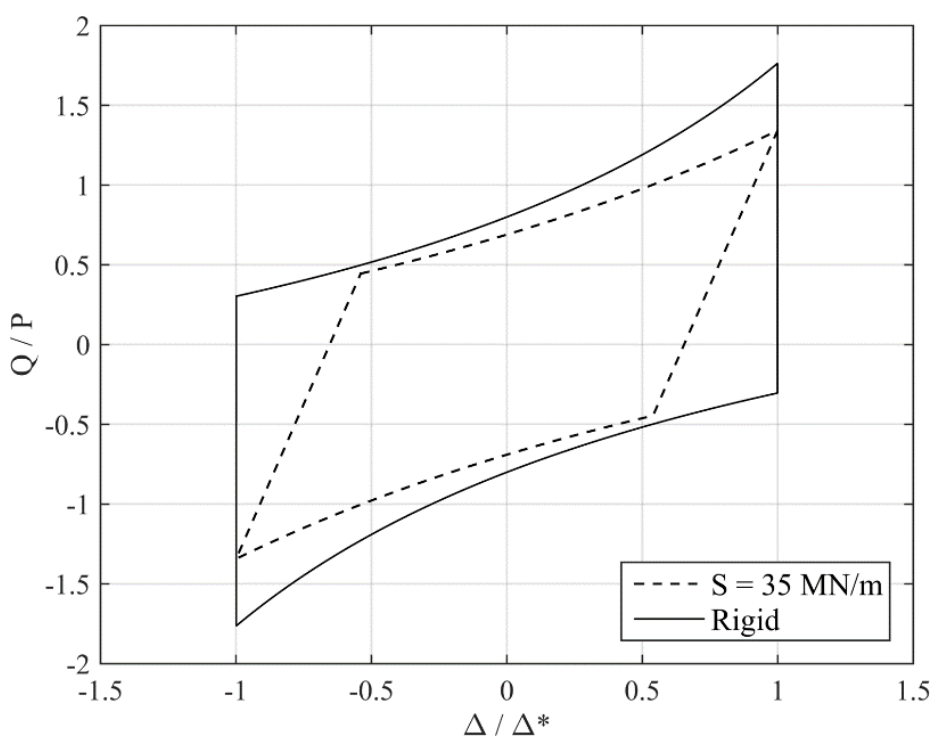

Figure 5 Comparison of the fretting loops from a rigid model and a model with a stiffness of $35 \mathrm{MN} / \mathbf{m}\left(\mathrm{x}_{\mathrm{o}}=\Delta^{*}=\right.$ $\left.50 \mu \mathrm{m}, \mathrm{P}=450 \mathrm{~N}, \mathrm{z}_{\mathrm{o}} / \mathrm{x}_{\mathrm{o}}=0.2, \mu=0.8\right)$.

Figure 5 shows a comparison of the models with rigid body and with a system stiffness of $35 \mathrm{MN} / \mathrm{m}$ under the applied displacement amplitude $\left(\Delta^{*}\right)$ of $50 \mu \mathrm{m}$. The $z_{o} / x_{o}$ value is chosen to be 0.2 as it is very close to the $D / W$ value of Example 2 (see Figure $3 b$ ). The rigid model fretting loop has been 
reported in the work of Mulvihill et al. [11]; this loop has vertical sides, which means the slip amplitude $\left(\delta^{*}\right)$ is the same as the displacement amplitude $\left(\Delta^{*}\right)$. The inclusion of the stiffness of the rig results in elastic deformation making up a fraction of the total applied displacement which results in the actual slip distance $(\delta)$ being smaller than the applied displacement $(\Delta)$. Due to the geometrical effects, the tractional force increases across the region of gross sliding for both cases; however, the maximum tractional force is lower when the system stiffness is included because of the smaller slip amplitude. In addition, it can also be seen that the dissipated energy (the area inside the fretting loop) is smaller.

It can also be observed that the top and the bottom of the fretting loops (which represent the gross sliding) are not symmetric about the mid-point of the slip, despite the groove itself being symmetric about that point. This is because the applied tractional force increases the contact force (and thus increases the frictional force itself) when $|\dot{\delta}| / \dot{\delta}$ has the same sign as $d z / d x$ (and decreases it when $|\dot{\delta}| / \dot{\delta}$ has the opposite sign to $d z / d x)$. Moreover, the magnitude of this change increases as the magnitude of $d z / d x$ increases (i.e. as the displacement from the centre of the groove increases). Therefore, the increase and decrease in tractional force either side of the mid-point of slip do not cancel each other out, which results in the increase in dissipated energy (compared to a Coulomb friction behaviour fretting loop) as reported in the work of Mulvihill et al. [11].

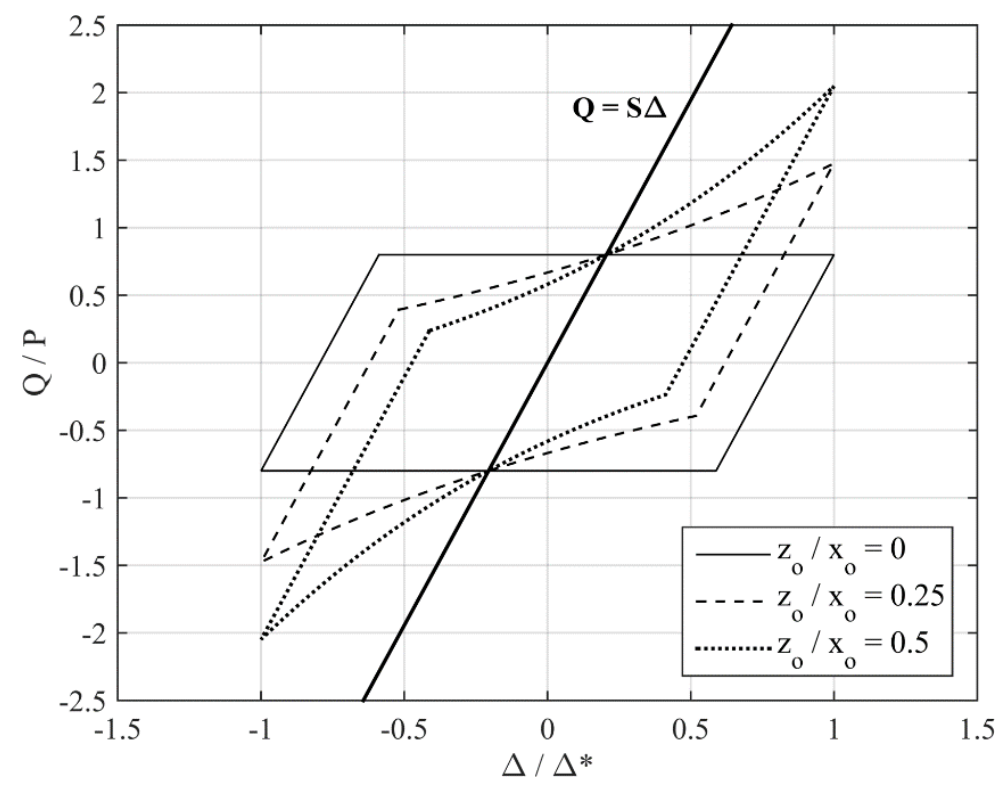

Figure 6 Comparison of fretting loops with grooves of different aspect ratios $\left(x_{o}=\Delta^{*}=50 \mu m, P=450 \mathrm{~N}, S=35\right.$ $\mathrm{MN} / \mathrm{m}, \boldsymbol{\mu}=\mathbf{0 . 8})$.

Figure 6 shows the comparison of fretting loops with different shapes of the groove (constant width, $\left(x_{o}\right)$ with different depths $\left(z_{o}\right)$ ). The displacement amplitude, the contact $\mathrm{CoF}$ and the system stiffness are the same in each case. It can be seen that both the slip amplitude and the variation of the tractional force during sliding are significantly influenced by the shape of the groove. As $z_{o} / x_{o}$ increases, the slip amplitude ( $\delta^{*}$ - which is equivalent to the loop semi-width when $Q / P=0$ ) is observed to get smaller and the tractional force varies in an increasingly non-linear manner with its peak value becoming larger. This behaviour is very similar to difference in fretting loops between Example 1 and Example 2 (Figure 3a), which indicates that in the case of Example 2, both the higher variation in 
tractional force and the reduction in slip amplitude are caused by geometric effects associated with a wear scar with a much higher depth to width ratio (Figure 3b).

\section{Difference between the contact coefficient of friction $(\mu)$ and the energy coefficient of friction $\left(\mu_{E}\right)$}

The analysis performed in the previous section shows the tractional force measured in a fretting test is made up of not only a frictional component but also a geometric component. In recent years, the ECoF method has been applied as a means of determining a representative $\mathrm{CoF}$ in cases where the tractional force was observed to vary across the region of slip. However, it can be seen from Figure 5 and Figure 6 that both the slip amplitude and the variation of the tractional force during sliding are affected by (i) the stiffness of the system and (ii) the geometry of the groove. The energy dissipated per cycle $\left(E_{d}\right)$, and thus the value of ECoF derived from it, are dependent on these two combined effects. Detailed analysis can be performed with the one-point contact analytical model to examine the effects of the $z_{o} / x_{o}$ value together with the stiffness of the system on $\operatorname{ECoF}\left(\mu_{E}\right)$ by comparing it with the real contact $\mathrm{CoF}(\mu)$. The dissipated energy per cycle can be calculated by integrating the tractional force with respect to $x$ for the whole fretting cycle. Since, the fretting loop is symmetric about the origin, the dissipated energy per cycle can be expressed as follows:

$$
E_{d}=2 \int_{x=-\delta^{*}}^{\delta^{*}} Q d x
$$

Note that this integral is with respect to $x$ over the range of the slip $(\delta)$ and not over the applied displacement $(\Delta)$ and thus is not readily visualised from the fretting loop.

On substituting Equation (9) into Equation (12):

$$
E_{d}=2 P \int_{x=-\delta^{*}}^{\delta^{*}} \frac{\mu+\left(\frac{2 z_{o}}{x_{o}^{2}} \cdot x\right)}{1-\mu\left(\frac{2 z_{o}}{x_{o}^{2}} \cdot x\right)} d x
$$

Integrating this yields,

$$
E_{d}=2 P \cdot\left[\left(-\frac{x_{o}^{2}}{2 z_{o}}-\frac{x_{o}^{2}}{2 \mu z_{o}}\right) \ln \left(1-\mu\left(\frac{2 z_{o}}{x_{o}^{2}} x\right)\right)-\frac{1}{\mu} x\right]_{x=-\delta^{*}}^{\delta^{*}}
$$

and thus:

$$
E_{d}=2 P \cdot\left\{\left(-\frac{x_{o}^{2}}{2 z_{o}}-\frac{x_{o}^{2}}{2 \mu z_{o}}\right)\left[\ln \left(1-\mu \frac{2 z_{o}}{x_{o}^{2}} \delta^{*}\right)-\ln \left(1+\mu \frac{2 z_{o}}{x_{o}^{2}} \delta^{*}\right)\right]-\frac{2}{\mu} \delta^{*}\right\}
$$

ECoF can therefore be calculated from Equation (1) as follows:

$$
\mu_{E}=\frac{E_{d}}{4 P \delta^{*}}=-\left(\frac{x_{o}^{2}}{4 z_{o} \delta^{*}}+\frac{x_{o}^{2}}{4 \mu z_{o} \delta^{*}}\right)\left[\ln \left(1-\mu \frac{2 z_{o}}{x_{o}^{2}} \delta^{*}\right)-\ln \left(1+\mu \frac{2 z_{o}}{x_{o}^{2}} \delta^{*}\right)\right]-\frac{1}{\mu}
$$


The displacement amplitude $\delta^{*}$ can be calculated from Equations (9) and (10) with the boundary condition that $x=\delta^{*}$ when $\Delta=\Delta^{*}$.

$$
\delta^{*}=\Delta^{*}-\frac{P}{S} \frac{\mu+\left(\frac{2 z_{o}}{x_{o}^{2}} \cdot \delta^{*}\right)}{\left(1-\mu\left(\frac{2 z_{o}}{x_{o}^{2}} \cdot \delta^{*}\right)\right)}
$$

(a)

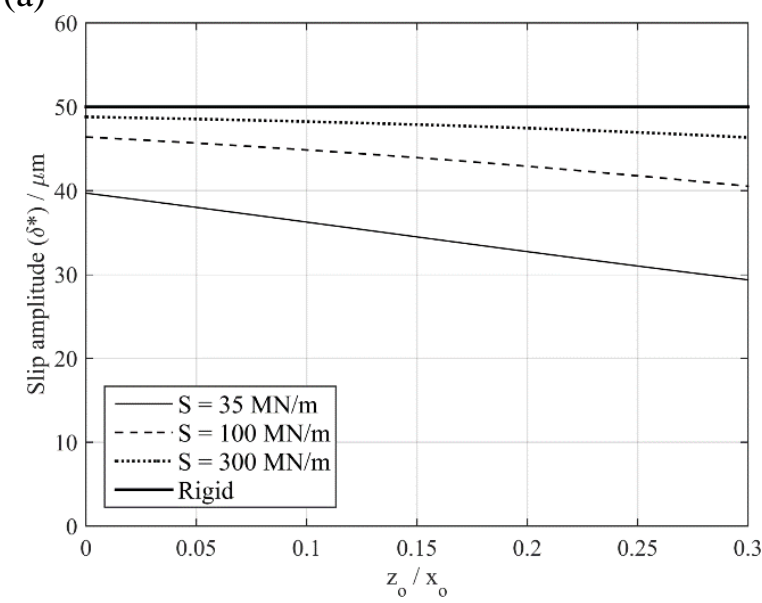

(b)

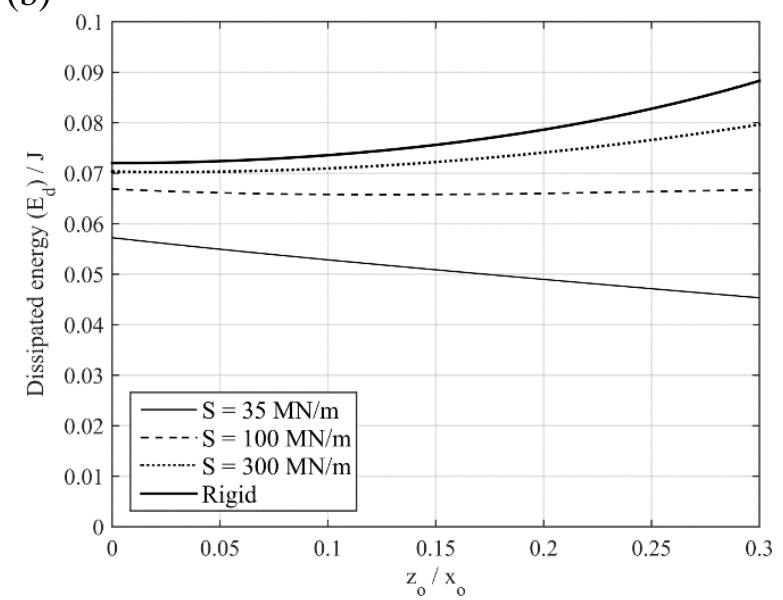

Figure 7 Effects of the groove aspect ratio $\left(z_{o} / x_{o}\right)$ and system stiffness $(S)$ on (a) slip amplitude and (b) dissipated energy per cycle $\left(x_{o}=\Delta^{*}=50 \mu m, P=450 \mathrm{~N}, \mu=0.8\right)$.

Figure 7a shows the effects of the $z_{o} / x_{o}$ ratio of the groove on the slip amplitude when a constant displacement amplitude is applied. It can be seen that when the system is rigid, the slip amplitude does not change with the $z_{o} / x_{o}$ ratio, and is equal to the displacement amplitude. However, when the system is not rigid, the slip amplitude decreases as the $z_{o} / x_{o}$ ratio increases, with the rate of decrease increasing as the system stiffness gets lower. Figure $7 \mathrm{~b}$ shows that the dissipated energy exhibits a more complex response; for a given value of $z_{o} / x_{o}$, a higher system stiffness always results in a higher dissipated energy. However, the dissipated energy increases with $z_{o} / x_{o}$ when the system stiffness is above a certain value and reduces with $z_{o} / x_{o}$ when the system stiffness is below that value. 


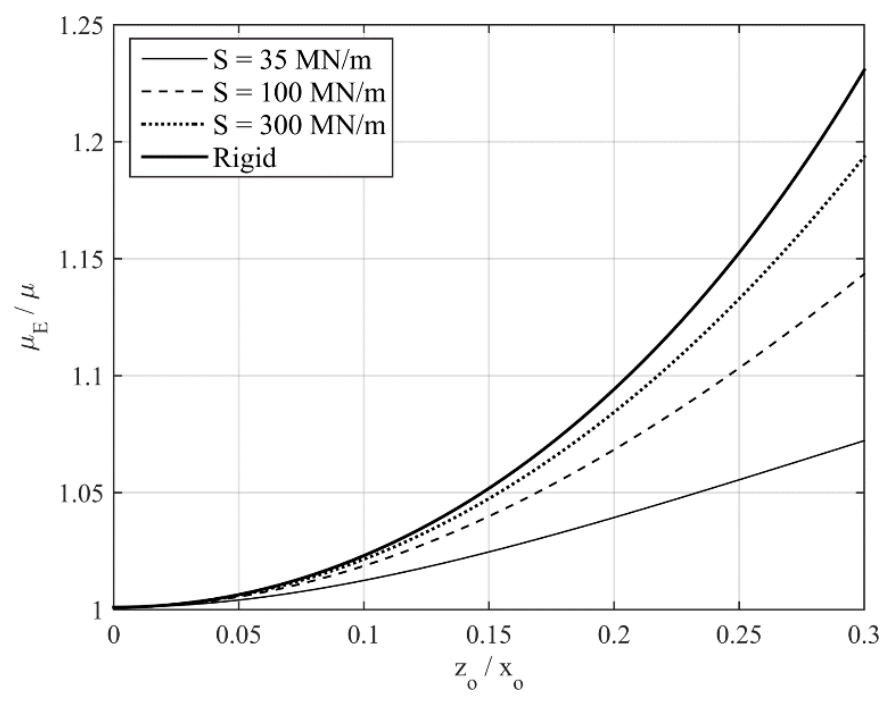

Figure 8 The effects of the groove aspect ratio $\left(z_{o} / x_{o}\right)$ and system stiffness (S) on the ratio of $(\mathrm{ECoF} / \mu)\left(x_{o}=\Delta^{*}=\right.$ $50 \mu m, P=450 \mathrm{~N}, \mu=0.8$ ).

Figure 8 shows the ratio of $\mu_{E} / \mu$ as a function of $z_{o} / x_{o}$ for a range of system stiffnesses, $S$; deviations from unity indicate the level to which $\mathrm{ECoF}\left(\mu_{E}\right)$ fails to accurately represent the true contact $\operatorname{CoF}(\mu)$. It can be seen that the ratio $\mu_{E} / \mu$ increases as both $z_{o} / x_{o}$ and $S$ increase. The increase in $\mu_{E} / \mu$ with $z_{o} / x_{o}$ is greater as the system stiffness increases since lower system stiffness results in a reduction in slip amplitude with increasing $z_{o} / x_{o}$ (see Figure 7a) which partly cancels out the effect of the rise in tractional force on ECoF. Under the conditions of Example 2 ( $S \approx 35 \mathrm{MN} / \mathrm{m}$, $z_{o} / x_{o} \approx 0.2$ (according to Figure $3 \mathrm{~b}$ )), it can be seen that $\mu_{E}$ is only around $4 \%$ higher than $\mu$. It should be noted that amongst the fretting wear scars observed following a wide range of tests performed at Nottingham, the scar of Example 2 has a particularly high aspect ratio (i.e. it results in a high value of $z_{o} / x_{o}$ ), which means most of other tests are expected to have difference between $\mu_{E}$ and $\mu$ of less than $4 \%$. However, it is also recognised that since $z_{o} / x_{o}$ may change during a fretting test, there may be changes in $\mathrm{ECoF}$ associated with that change which may be misinterpreted as changes in the true contact $\mathrm{CoF}(\mu)$.

\section{A new method to derive the coefficient of friction}

It has been demonstrated in the previous section that the $\mathrm{ECoF}$ method cannot entirely take account of the geometric component in the tractional force, and thus results in a value of $\operatorname{CoF}\left(\mu_{E}\right)$ which is not the true contact $\operatorname{CoF}(\mu)$. In light of this discrepancy, a new method is proposed here to derive the true contact $\mathrm{CoF}$ from the fretting loops which is not influenced by the geometrical developments of the contact surfaces. It can be seen from Figure 6 that the fretting loops with different groove shapes all intersect at two points, at which the tractional force is equal to the product of the normal load $(P)$ and the true contact $\mathrm{CoF}(\mu)$. This relates to the point when the round-ended pad passes the centre of the groove (i.e. the point of zero gradient). This can be demonstrated by substituting $Q=\mu P$ into Equation (8):

$$
\mu P=P \frac{\frac{|\dot{\delta}|}{\dot{\delta}} \mu+\frac{2 z_{o}}{x_{o}^{2}} \cdot x}{1-\frac{|\dot{\delta}|}{\dot{\delta}} \mu \frac{2 z_{o}}{x_{o}^{2}} \cdot x} \Rightarrow x=0
$$


Substitute $x=0$ into Equation (10):

$$
0=\Delta-\frac{Q}{S} \Rightarrow Q=S \Delta
$$

As such, the true contact $\mathrm{CoF}$ is defined as the ratio of $Q / P$ at the point where the slope of the contact itself is zero; this point in the fretting loop is that which also lies on the on the line $Q=S \Delta$, as indicated in Figure 6. By applying this method to each fretting loop throughout a fretting test, the evolution of the true contact $\mathrm{CoF}$ can be evaluated (this will be termed the geometry-independent coefficient of friction (GICoF)).

(a)

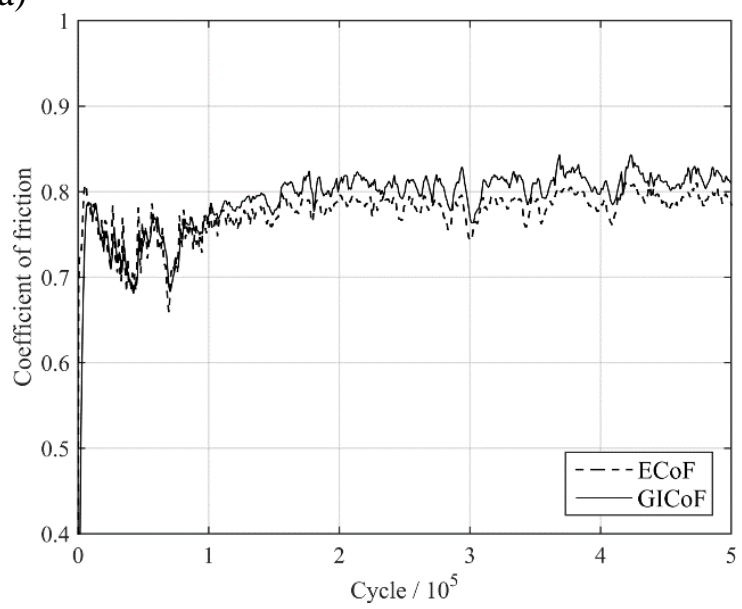

(b)

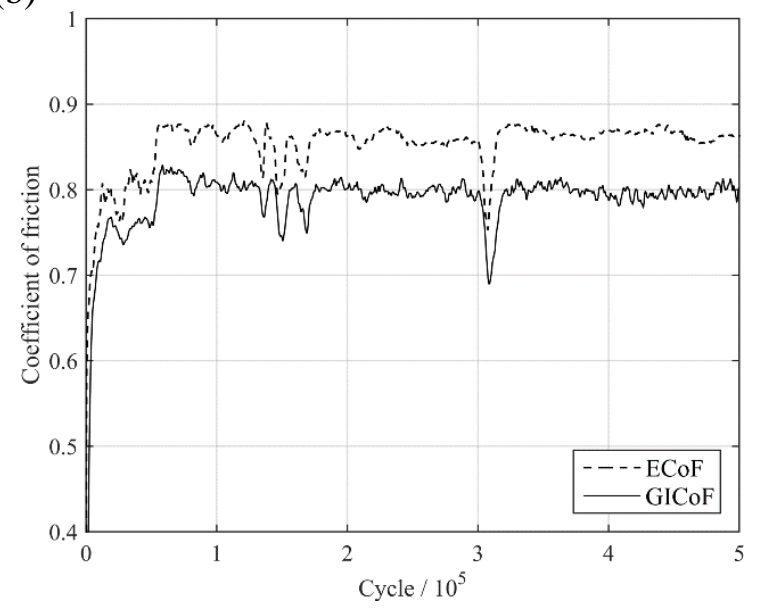

Figure 9 Comparison of the evolution of ECoF and GICoF for the two tests presented in Figure 3: (a) Example 1 (b) Example 2.

Figure 9 shows the comparison of applying the ECoF and GICoF method for both Example 1 (a) and Example 2 (b). It can be seen that for Example 1, both of the methods give very similar outcome in terms of the CoF. However, for Example 2, the value derived by the ECoF method is clearly higher than that derived by the GICoF (more than $5 \%$ for about $80 \%$ of the test). This is in accord with the conclusions drawn in the previous sections, namely that the geometry in Example 2 exerts a significant influence on tractional force in light of its high aspect ratio. It should also be noted that ECoF and GICoF are very close at the early stage of Example 2 (i.e. almost the same at the beginning of the test) when the wear scar itself is less fully developed. Furthermore, it should be noted that the application of the GICoF method does not require any analysis of the shape of the wear scar, and can be derived simply from the fretting loop alone. 
(a)

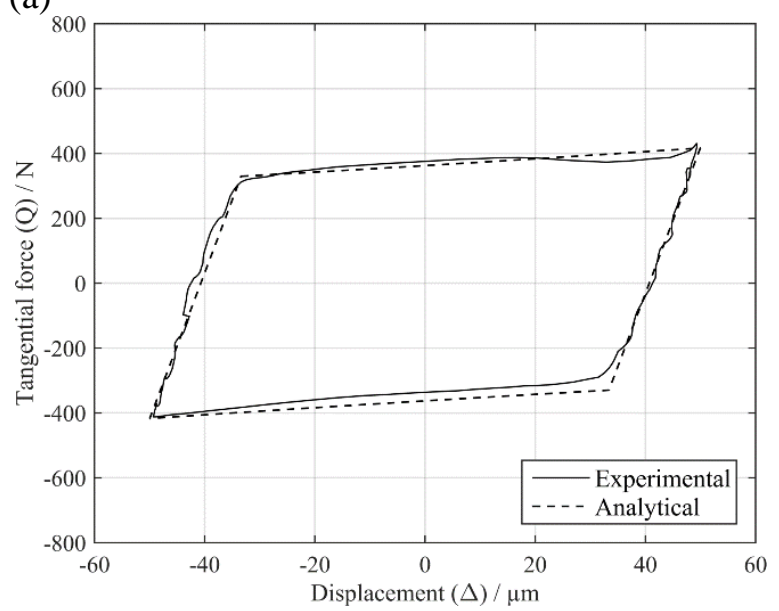

(b)

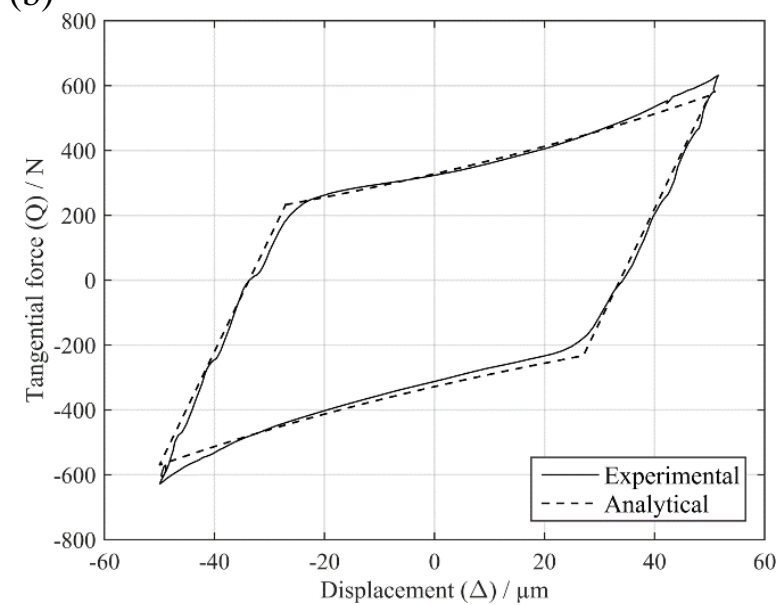

Figure 10 Comparison of the experimentally measured and modelled fretting loops for (a) Example 1, (b) Example 2. The measured loops are the final loops in the respective tests.

Validations of the one-point contact analytical model, the conversion method (from a real wear scar to a groove shape in the model) and application of the GICoF method have been performed by comparing the modelled fretting loops with the measured ones for both Example 1 and Example 2. In the converted one-point contact models, the widths of the grooves are set to be equal to $\Delta^{*}(50 \mu \mathrm{m})$ for both cases; and the depth of the corresponding groove $\left(z_{o}\right)$ is calculated to be $1.8 \mu \mathrm{m}$ for Example 1 and $8.0 \mu \mathrm{m}$ for Example 2. The value of the contact $\operatorname{CoF}(\mu)$ are set to be equal to the GICoF obtained from the measured loops ( 0.825 for both cases). The system stiffnesses $(S)$ are applied according to the measured loops to be $45 \mathrm{MN} / \mathrm{m}$ for Example 1 and $35 \mathrm{MN} / \mathrm{m}$ for Example 2. Figure 10 shows the comparisons of the measured and modelled fretting loops for both tests. Good agreements have been obtained for both cases, providing confidence in the validity of both the analytical model and the GICoF method.

\section{Discussion}

The difference in the measured fretting loops of Example 1 and Example 2 (Figure 3a) shows that the fretting loop shape can be very different although the same normal load and displacement amplitude are applied. A wear scar of the type presented in Example 2 will result in a higher variation in tractional force; the higher peak tractional force will result in higher elastic deformation of the whole system which accounts for a larger part of the applied displacement thus reduces the slip amplitude (see Figure 6). Since most experimental fretting rigs reported in the literature control the displacement amplitude rather than the slip amplitude; it is therefore necessary to include the system stiffness into such analysis. Compared to the fretting loop derived from a rigid interaction model, a significant difference can be observed in the tractional force and slip amplitude when same displacement amplitude is applied (see Figure 5).

In research reported in the literature, the ECoF method has been widely applied in an attempt to address variations in the tractional force over the region of slip in a fretting loop. Although it has been effective in addressing the high peak tractional force effects, it has been shown here that $\mu_{E}$ (derived from the ECoF method) is somewhat dependent upon both scar geometry and system stiffness (rather than independent of these as would be desired). To eliminate fully the geometric influence on derived values of CoF, the GICoF method has been introduced. 
(a)

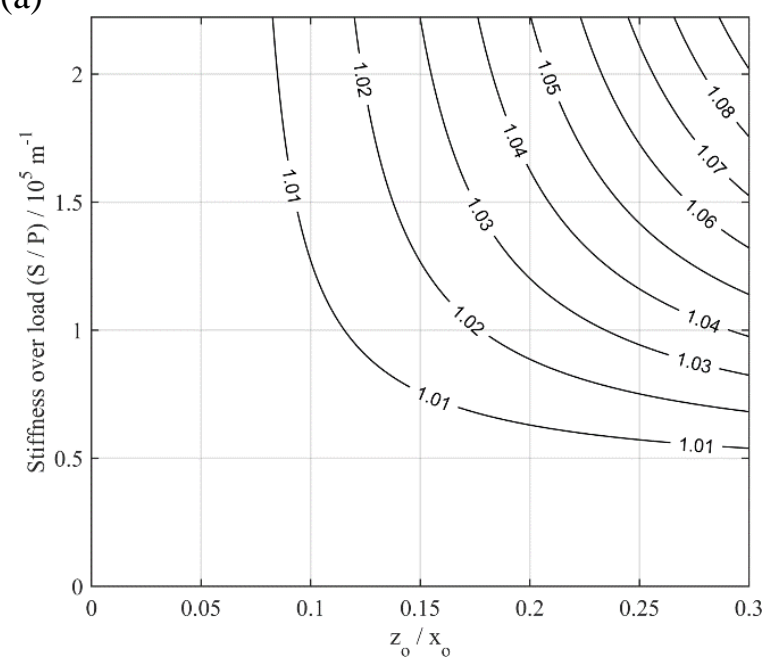

(b)

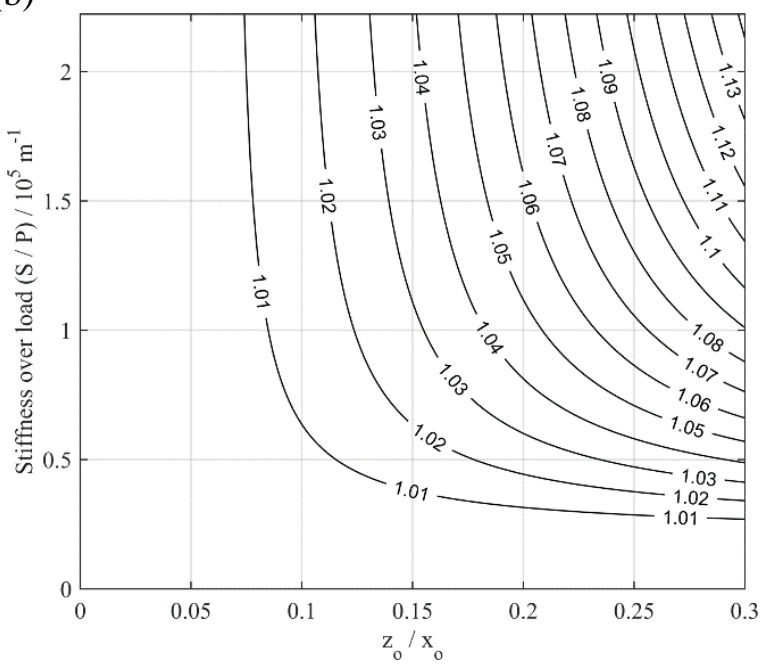

Figure 11 Effects of both the stiffness of the system $(S)$ and the depth over width ratio $\left(z_{0} / x_{0}\right)$ on the ratio of $\left(\mu_{E} / \mu\right)$ for (a) $\Delta^{*}=25 \mu \mathrm{m}\left(\right.$ b) $\Delta^{*}=50 \mu \mathrm{m}(\mu=0.8)$. Contours of $\mu_{\mathrm{E}} / \mu$ are plotted with the values indicated within the contours themselves.

Whilst it is recommended that the GICoF method should be used in future for analysis of fretting loops, it is also recognized that many data already in the literature have been processed via the ECoF method. As such, it is appropriate to examine the magnitude of differences between the coefficients derived via the two methods. The combined effects of the system stiffness $(S)$ and the value of $z_{o} / x_{o}$ on the ratio of $\mu_{E} / \mu$ are plotted in Figure 11, in the form of $\mu_{E} / \mu$ contours. It can be seen from Equation (15) that the ECoF is only influenced by the slip amplitude $\left(\delta^{*}\right)$ when $z_{o}, x_{o}$, and $\mu$ are constants. Furthermore, the slip amplitude is dependent on $P / S$ when constant $\Delta^{*}$ is applied (see Equation (16)). Therefore, the ratio of the stiffness to the applied load $(S / P)$ is utilised in the maps to represent the system stiffness effects. Figure 11 shows the cases when $\Delta^{*}=25 \mu m$ (Figure 11a) and $\Delta^{*}=50 \mu \mathrm{m}$ (Figure 11b), indicating that the contours are also dependent upon applied displacement amplitude. These contours allow an assessment to be made as to the significance of the difference between $\mu_{E}$ and $\mu$. It can be seen that the differences increase with increases in $z_{o} / x_{o}, S / P$ and $\Delta^{*}$. The maps in Figure 11 cover regions which are representative of tests conducted at the University of Nottingham, and variations of up to $13 \%$ are seen in the most extreme cases. However, it must also be recognized that the majority of tests reside in the region where the difference between $\mu_{E}$ and $\mu$ is less than $5 \%$.

\section{Conclusions}

An analytical model (based on Mulvihill's wear scar interaction model) has been developed to analyse the interactive effects of the stiffness of the system $(S)$ and the wear scar geometry on the tractional forces measured in experimental fretting tests. The results show that both the system stiffness and the shape of scar can have significant influences on (i) the variation of the gross sliding tractional force and (ii) the slip amplitude, when constant displacement amplitude is applied (most of the fretting tests are performed under this condition). These effects can cause the $\mathrm{ECoF}\left(\mu_{E}\right)$ to be different from the real contact $\mathrm{CoF}(\mu)$. However, this difference is typically (under conditions representative of the tests conducted at the University of Nottingham) expected to be less than 5\%. To eliminate better the geometric component on the $\mathrm{CoF}$, the GICoF method is introduced to calculate the contact $\mathrm{CoF}(\mu)$ as the ratio of $Q / P$ where the contact itself is at the point of zero slope. 


\section{Acknowledgements}

The authors would like to thank the University of Nottingham for the award of the "Dean of Engineering Research Scholarship for International Excellence" to Xiaozhe Jin.

\section{References}

[1] O. Vingsbo, S. Söderberg, On fretting maps, Wear. 126 (1988) 131-147. doi:10.1016/00431648(88)90134-2.

[2] S. Fouvry, T. Liskiewicz, P. Kapsa, S. Hannel, E. Sauger, An energy description of wear mechanisms and its applications to oscillating sliding contacts, Wear. 255 (2003) 287-298. doi:10.1016/S0043-1648(03)00117-0.

[3] C.A. Coulomb, Theorie des machines simples: en ayant egard au frottement de leurs parties et a la roideur des cordages, Bachelier, 1821.

[4] R. Magaziner, O. Jin, S. Mall, Slip regime explanation of observed size effects in fretting, Wear. 257 (2004) 190-197. doi:10.1016/j.wear.2003.12.005.

[5] H. Lee, S. Mall, Fretting behavior of shot peened Ti-6Al-4V under slip controlled mode, Wear. 260 (2006) 642-651. doi:10.1016/j.wear.2005.03.022.

[6] N.M. Everitt, J. Ding, G. Bandak, P.H. Shipway, S.B. Leen, E.J. Williams, Characterisation of fretting-induced wear debris for Ti-6Al-4 V, Wear. 267 (2009) 283-291. doi:10.1016/j.wear.2008.12.032.

[7] S. Fouvry, P. Kapsa, H. Zahouani, L. Vincent, Wear analysis in fretting of hard coatings through a dissipated energy concept, Wear. 203-204 (1997) 393-403. doi:10.1016/S00431648(96)07436-4.

[8] C. Paulin, S. Fouvry, C. Meunier, Finite element modelling of fretting wear surface evolution: Application to a Ti-6A1-4V contact, Wear. 264 (2008) 26-36. doi:10.1016/j.wear.2007.01.037.

[9] S. Fouvry, P. Duó, P. Perruchaut, A quantitative approach of Ti-6Al-4V fretting damage: friction, wear and crack nucleation, Wear. 257 (2004) 916-929. doi:10.1016/j.wear.2004.05.011.

[10] T. Dick, C. Paulin, G. Cailletaud, S. Fouvry, Experimental and numerical analysis of local and global plastic behaviour in fretting wear, Tribol. Int. 39 (2006) 1036-1044. doi:10.1016/j.triboint.2006.02.023.

[11] D.M. Mulvihill, M.E. Kartal, A.V. Olver, D. Nowell, D.A. Hills, Investigation of nonCoulomb friction behaviour in reciprocating sliding, Wear. 271 (2011) 802-816. doi:10.1016/j.wear.2011.03.014.

[12] J. Hintikka, A. Lehtovaara, A. Mäntylä, Normal displacements in non-Coulomb friction conditions during fretting, Tribol. Int. 94 (2016) 633-639. doi:10.1016/j.triboint.2015.10.029.

[13] S.R. Pearson, P.H. Shipway, Is the wear coefficient dependent upon slip amplitude in fretting? Vingsbo and Söderberg revisited, Wear. 330-331 (2014) 93-102. doi:10.1016/j.wear.2014.11.005.

[14] A.R. Warmuth, P.H. Shipway, W. Sun, Fretting wear mapping: the influence of contact geometry and frequency on debris formation and ejection for a steel-on-steel pair, Proc. R. Soc. London A Math. Phys. Eng. Sci. 471 (2015). doi:10.1098/rspa.2014.0291. 